\title{
Dihydropyrimidinase-related protein 5 controls glioblastoma stem cell characteristics as a biomarker of proneural-subtype glioblastoma stem cells
}

\author{
MIN GI PARK ${ }^{1 *}$, SUNYOUNG SEO ${ }^{1,2^{*}}$, SEOK WON HAM ${ }^{1,2}$, SANG-HUN CHOI $^{1,2}$ and HYUNGGEE KIM ${ }^{1,2}$ \\ ${ }^{1}$ Department of Biotechnology, College of Life Sciences and Biotechnology; \\ ${ }^{2}$ Institute of Animal Molecular Biotechnology, Korea University, Seoul 02841, Republic of Korea
}

Received November 5, 2019; Accepted April 15, 2020

DOI: $10.3892 / 01.2020 .11668$

\begin{abstract}
Glioblastoma (GBM) is the most aggressive and malignant brain tumor, resulting in a poor prognosis. The current therapy for GBM consists in concurrent radiation and chemotherapy following removal of the tumor. Although the therapy prolongs patient survival, recurrence often occurs. The major cause of tumor recurrence is thought to be GBM stem cells (GSCs), which aid the development of chemo-radiotherapy resistance, and can self-renew and aberrantly differentiate. Therefore, GSCs should be targeted to eradicate the tumor and prevent recurrence. Transcriptomic analysis has categorized GBM into proneural (PN), mesenchymal and classical subtypes, and the outcome of recurrence and prognosis markedly depends on subtype. To identify specific GSC markers, the present study analyzed public microarray and RNA-seq data and identified dihydropyrimidinase-related protein 5 (DRP5) as a candidate GSC marker. DRP5 is known to mediate semaphorin $3 \mathrm{~A}$ signaling and is involved in the regulation of neurite outgrowth and axon guidance during neuronal development. In the present study, DRP5 was specifically upregulated in the PN-subtype GSCs and served crucial roles in maintaining GSC properties, including tumor sphere formation, stem cell marker expression and xenograft tumor growth. Furthermore, bioinformatics analysis revealed that DRP5 expression was positively correlated with signatures of stemness, including Notch, Hedgehog and Wnt/ $\beta$-catenin expression, which are also known to be positively correlated with PN-subtype gene signatures. Conversely, DRP5
\end{abstract}

Correspondence to: Dr Hyunggee Kim, Department of Biotechnology, College of Life Sciences and Biotechnology, Korea University, 145 Anam-ro, Seongbuk-gu, Seoul 02841, Republic of Korea

E-mail: hg-kim@korea.ac.kr

"Contributed equally

Key words: glioblastoma, glioblastoma stem cell, dihydropyrimidinase-related protein 5 , stemness, proneural subtype biomarker expression was negatively correlated with $\mathrm{NF}-\kappa \mathrm{B}$ and signal transducer and activator of transcription 3 stemness signatures, which are negatively correlated with PN-subtype gene signatures. Taken together, these findings suggested that DRP5 was specifically expressed in PN-subtype GSCs and may be used as a functional marker of PN-subtype GSCs.

\section{Introduction}

Glioblastoma (GBM) is the most incurable primary brain tumor, with the median survival of patients with GBM being 12-14 months (1). The 5-year survival rate was $28 \%$ in patients diagnosed between 1985 and 2005, and it is estimated that 138,054 patients had a GBM diagnosis in the United States in $2010(2,3)$. Therapies targeting glioblastoma are limited, resulting in poor patient prognosis (3). The standard therapy is the combination of radiation and chemotherapy using temozolomide following surgical resection of the tumor. However, despite treatment, patients often experience tumor recurrence, leading to poor survival rates (4). Cancer stem cells (CSCs) are considered as a major cause of tumor relapse and malignancy (5). CSC characteristics include their self-renewal capacity, persistent proliferation and tumorigenicity when orthotopically injected into immunodeficient mice (6,7). GBM stem cells (GSCs) with a phenotype of resistance to chemotherapy are present in low number in tumor bulks and are considered as a major cause of recurrence following standard therapy (8). GSC-targeted therapy has decreased the recurrence rate and improved tumor clearance (9-11). Recently, based on transcriptomic profiling, GBM has been classified into the proneural (PN), mesenchymal (MES) and classical subtypes (12). The outcome of therapeutic approaches and prognosis of patients is associated with the GBM subtype, and patients with MES GBM have a poorer prognosis than those with non-MES GBM due to the tumor immune microenvironment (12). Therefore, defining the subtype of GBM and targeting GSCs are crucial for successful therapy.

The dihydropyrimidinase-related protein (DRP) family, also known as collapsin-response mediator proteins, was first identified as a cytosolic protein family that mediates neurite outgrowth, growth cone collapse and axon guidance via semaphorin 3A signaling during neuronal development (13-15). The DRP family is composed of five members (DRP1-5). 
DRP1 overexpression decreases invasion in lung cancer (16). Furthermore, inhibition of DRP2 phosphorylation reportedly enhances paclitaxel on-target activity in ovarian cancer (17). In addition, inhibition of DRP3 promotes lung cancer metastasis. Also, DRP4 is induced by TP53 and regulates energy metabolism in adipocytes and non-small cell lung cancer $(18,19)$. DRP5 is a recently classified member of the DRP protein family that is expressed in neuroendocrine lung cancer and glioblastoma (16). DRP5 can stabilize Notch receptors and promote Notch signaling by preventing ubiquitination and lysosomal degradation of Notch receptors, contributing therefore to cell proliferation in GBM (20). Furthermore, DRP5 expression is associated with cancer cell growth rate and tumorigenicity in osteosarcoma, and with neurological autoimmune disease $(21,22)$.

Overall, defining the GBM subtype and targeting GSCs is crucial for a successful therapy. Therefore, functional markers for each subtype-specific GSCs should be defined. Public gene expression databases are useful for finding candidate genes that may serve as GSC markers. The present study analyzed these databases to identify specific functional markers of GSCs.

\section{Materials and methods}

Cells and cell culture conditions. The human GBM cell lines U87MG (RRID, CVCL_0022, glioblastoma of unknown origin), LN229 (RRID, CVCL_0393), LN18 (RRID, CVCL_0392), T98G (RRID, CVCL_0556), A172 (RRID, CVCL_0131) and A1207 (RRID, CVCL_8481) were purchased from the American Type Culture Collection. Normal human astrocytes (NHAs) were purchased from ScienCell Research Laboratories, Inc. Human GBM cells and NHAs were cultured in high-glucose DMEM supplemented with 10\% FBS, $1 \%$ penicillin/streptomycin and $2 \mathrm{mM}$ L-glutamine (all from HyClone; GE Healthcare Life Sciences) and placed at $37^{\circ} \mathrm{C}$ in a humidified incubator containing $5 \% \mathrm{CO}_{2}$ and $95 \%$ humidity. PN-GSCs [528NS, GSC11 (RRID, CVCL_DR55) and GSC23 (RRID, CVCL_DR59)], MES-GSCs (GSC20) and non-defined GSCs (GSC28) were kindly provided by Dr Ichiro Nakano of Ohio State University (528NS) and Dr Erik Sulman of The University of Texas M.D. Anderson Cancer Center (GSC11, GSC23, GSC20 and GSC28). GSCs were cultured in DMEM/F12 (HyClone; GE Healthcare Life Sciences) supplemented with $0.04 \%$ B27 (Invitrogen; Thermo Fisher Scientific, Inc.), $20 \mathrm{ng} / \mathrm{ml}$ epidermal growth factor (EGF; R\&D Systems, Inc.), $20 \mathrm{ng} / \mathrm{ml}$ basic fibroblast growth factor (bFGF; R\&D Systems, Inc.), $1 \%$ penicillin/streptomycin (HyClone; GE Healthcare Life Sciences) and $2 \mathrm{mM}$ L-glutamine (HyClone; GE Healthcare Life Sciences) and placed at $37^{\circ} \mathrm{C}$ in a humidified incubator containing 5\% $\mathrm{CO}_{2}$ and $95 \%$ humidity. Mycoplasma testing was performed using reverse transcription-quantitative PCR (RT-qPCR). All human cell lines were authenticated using short tandem repeat profiling.

Plasmids, transfection and lentivirus infection. The DRP5 short hairpin RNA (shRNA) target sequence 5'-CAGGAC TCACTGTCCAATCTAC-3' was selected and screened for off-target complementarity using NCBI-Blast (https://blast. ncbi.nlm.nih.gov/Blast.cgi). The shRNA was cloned into the commercial pLKO.1-puro lentiviral vector (Sigma-Aldrich; Merck KGaA). A total of $2 \times 10^{6} 293 \mathrm{FT}$ cells (Invitrogen; Thermo Fisher Scientific, Inc.) were seeded on 100Ф cell culture plates for transfection. After $24 \mathrm{~h}, 4 \mu \mathrm{g}$ pLKO.1-shNT-puro, $4 \mu \mathrm{g}$ pLKO.1-shDRP5-puro and second-generation lentiviral packaging vectors ( $3 \mu \mathrm{g}$ pCMV-dR8.91 and $1 \mu \mathrm{g} \mathrm{VSV-G})$ were transfected into 293FT cells using Lipojet ${ }^{\mathrm{TM}}$ in vitro transfection reagent (SignaGen Laboratories) to produce lentiviral particles. Lentiviruses were concentrated using the Lenti- $\mathrm{X}^{\mathrm{TM}}$ Concentrator (Takara Bio, Inc.) and resuspended into $400 \mu \mathrm{l}$ PBS. A total of $1.5 \times 10^{6} 528 \mathrm{NS}$ cells were seeded on $100 \Phi$ cell culture plates for infection. After 24 h, 528NS cells were infected with $200 \mu \mathrm{l}$ lentiviral particles. Cells infected with the lentiviral particles were selected with Puromycin $(3 \mu \mathrm{g} / \mathrm{ml})$ during a 1-week incubation. Subsequently, pLKO.1-shNT-puro lentivirus-infected 528NS cells were renamed 528NS-puro and pLKO.1-shDRP5-puro lentivirus-infected 528NS cells were renamed 528NS-DRP5 knockdown (KD). Knockdown efficiency was confirmed by western blotting.

Western blotting. Cells from the aforementioned cell lines were lysed using RIPA lysis buffer (150 mM sodium chloride, $1 \%$ NP-40, $0.1 \%$ SDS and $50 \mathrm{mM}$ Tris $\mathrm{pH} 7.4$ ) containing $1 \mathrm{mM} \beta$-glycerophosphate, $2.5 \mathrm{mM}$ sodium pyrophosphate, $1 \mathrm{mM}$ sodium fluoride, $1 \mathrm{mM}$ sodium orthovanadate and protease inhibitor (Roche Diagnostics). Cell lysis was performed through two-time sonication (one cycle sonication condition, $20 \mathrm{kHz}$; amplitude $20 \%$; $3 \mathrm{sec}$ on, $2 \mathrm{sec}$ off, total $15 \mathrm{sec} ; 4^{\circ} \mathrm{C}$; total energy input, $8 \mathrm{~J}$ ) and the lysed cells were incubated at $4^{\circ} \mathrm{C}$ for $3 \mathrm{~h}$ and centrifuged at $21,000 \mathrm{x} \mathrm{g}$ at $4^{\circ} \mathrm{C}$ for $20 \mathrm{~min}$ to obtain the supernatant. Total protein concentration was quantified using Bradford assay reagent (Bio-Rad Laboratories, Inc.) according to the manufacturer's protocol. A total of $10 \mu \mathrm{g}$ protein/lane was separated by $10 \%$ SDS-PAGE and transferred onto polyvinylidene fluoride membranes (Pall Life Sciences). Membranes were blocked with $5 \%$ non-fat milk for $1 \mathrm{~h}$ at $25^{\circ} \mathrm{C}$ and incubated for $12 \mathrm{~h}$ at $4^{\circ} \mathrm{C}$ with either rabbit anti-DRP5 (1:500; cat. no. HPA072387; Atlas Antibodies) or mouse anti- $\beta$-actin (1:10,000; cat. no. A5316; Sigma-Aldrich; Merck KGaA). Membranes were subsequently incubated for $2 \mathrm{~h}$ at $25^{\circ} \mathrm{C}$ with horseradish peroxidase-conjugated goat anti-rabbit (cat. no. 31460) or anti-mouse (cat. no. 31430) IgG secondary antibodies (both 1:5,000; Pierce; Thermo Fisher Scientific, Inc.), and protein bands were visualized using the SuperSignal West Pico Chemiluminescent Substrate (Pierce; Thermo Fisher Scientific, Inc.).

In vitro limiting dilution assay (LDA). 528NS cells infected with pLKO.1-puro (control) or pLKO.1-shDRP5-873 lentivirus were plated in 96-well plates with a decreasing number (20, 10, 5 and 1) cells/well, with 24 wells used for each cell number. The cells were cultured in DMEM/F12 supplemented with $0.2 \%$ B27, $20 \mathrm{ng} / \mathrm{ml} \mathrm{bFGF}$ and $20 \mathrm{ng} / \mathrm{ml}$ EGF. The medium was replaced every 3 days with fresh bFGF and EGF. Neurospheres were counted after 13 days using a light microscope (CKX53; Olympus Corporation). The experiment was performed in duplicate. Extreme limiting dilution analysis was performed using the ELDA software (http://bioinf.wehi. edu.au/software/elda/). 
Orthotopic glioma cell implantation. Ten female BALB/c nude mice (4-5 weeks old; average weight, $15 \mathrm{~g}$ ), were purchased through Orient Bio, Inc. Mice were maintained in a 12 -h light/12-h dark cycle at $23 \pm 2^{\circ} \mathrm{C}$ and $55 \pm 5 \%$ humidity, and they had constant access to food and water. For orthotopic implantation, cells were resuspended in PBS, and $3 \mu 1$ of $5 \times 10^{4}$ 528NS-puro and 528NS-DRP5 KD cells were stereotactically injected into the brains of $5 \mathrm{BALB} / \mathrm{c}$ nude mice each (coordinates, $2 \mathrm{~mm}$ right and $1 \mathrm{~mm}$ rostral from bregma, and $3 \mathrm{~mm}$ depth from the surface of the skull) as previously described (23). Anesthesia was performed using Zoletil ${ }^{\circledR}$ (30 mg/kg) and Rompun ${ }^{\circledR}(10 \mathrm{mg} / \mathrm{kg})$.

Mice generally exhibited neurological problems after 35-40 days. Therefore, after 40 days mice were anesthetized by intraperitoneal injection of Avertin $(250 \mathrm{mg} / \mathrm{kg}$; Sigma-Aldrich; Merck KGaA). Once mice lost consciousness, their body temperature was determined using an infrared thermometer. When body temperature decreased by $>2{ }^{\circ} \mathrm{C}$ compared, mice were perfused with PBS and $4 \%$ paraformaldehyde (PFA; Sigma-Aldrich; Merck KGaA) for tissue fixation $\left(48 \mathrm{~h}\right.$ at $\left.4^{\circ} \mathrm{C}\right)$. Anesthetized mice were subsequently decapitated prior to brain collection. Brain was cut into two pieces and stored in 4\% PFA until paraffin blocks were made. Animal experiments were approved by the Korea University Institutional Animal Care \& Use Committee and performed according to the governmental and institutional guidelines and Korean regulations (approval no. KUIACUC-2018-0017).

Hematoxylin and eosin $(H \& E)$ staining. Fixed brain tissues were embedded in paraffin, sectioned into 4- $\mu \mathrm{m}$ thick slides and mounted on glass slides. For deparaffinization, slides were incubated at $65^{\circ} \mathrm{C}$ in a dry oven for $20 \mathrm{~min}$. For hydration, the slides were serially dipped into xylene, absolute ethanol, 95, 80 and $70 \%$ ethanol and washed with distilled water. After deparaffinization and hydration, slides were stained with hematoxylin (Merck KGaA) for 5 min and rinsed with tap water. Subsequently, tissue slides were dipped 10-15 times in acidic alcohol and rinsed with tap water. All slides were stained with an eosin solution (Merck KGaA) for $30 \mathrm{sec}$, followed by washing with distilled water. Finally, the stained slides were serially dehydrated with $95 \%$ ethanol, absolute ethanol and xylene, and mounted with mounting solution (SP15-100; Fisher Chemical; Thermo Fisher Scientific, Inc.). Tumor area was captured and quantified by measuring the pixel intensity of the stained tumor area using a dissecting light microscope (magnification, x10) and ImageJ software (v1.8.0_112; National Institutes of Health). Only four slides per group were analyzed due to the loss of one sample. After measurement, the mean of DRP5-KD pixels was normalized to the mean of control pixels.

Immunofluorescence. A total of $5 \times 10^{4}$ cells/well (528NS, GSC11, A172 and NHA cells) were seeded on cover slips in a 4-well plate. After $24 \mathrm{~h}$, cells were washed with ice-cold PBS and fixed with $4 \%$ PFA for $20 \mathrm{~min}$ at $25^{\circ} \mathrm{C}$, followed by permeabilization with $0.3 \%$ Triton $\mathrm{X}-100$ in PBS and blocking with $3 \%$ bovine serum albumin for $1 \mathrm{~h}$ at $25^{\circ} \mathrm{C}$. The cells were stained with DRP5 antibody (1:100; cat. no. HPA072387; Atlas Antibodies) for $12 \mathrm{~h}$ at $4^{\circ} \mathrm{C}$. Cells were washed with PBS three times for 5 min each and incubated with Alexa 594-conjugated secondary antibody (1:500; cat. no. A37240; Invitrogen; Thermo Fisher Scientific, Inc.) at $25^{\circ} \mathrm{C}$ for $2 \mathrm{~h}$. After DAPI staining (Sigma-Aldrich; Merck KGaA) for $10 \mathrm{~min}$ at $25^{\circ} \mathrm{C}$, slides were washed three times with PBS, mounted in ProLong ${ }^{\mathrm{TM}}$ Gold Antifade Mountant (P36930; Invitrogen; Thermo Fisher Scientific, Inc.), and stored at $4^{\circ} \mathrm{C}$ in the dark. Fluorescence was detected using a confocal microscope (magnification, $\mathrm{x} 40$ ).

$R T-q P C R$. RT-qPCR was performed to quantify mRNA levels. Total RNA was isolated from cells (NHA, 528NS, GSC11, GSC23, GSC20, GSC28, U87MG, T98G, LN18, LN229, A1207, A172, 528NS-puro and 528NS-shDRP5 KD) using TRIzol ${ }^{\circledR}$ reagent (Invitrogen; Thermo Fisher Scientific, Inc.) according to the manufacturer's protocol. A total of $1 \mu \mathrm{g}$ RNA pre-treated with RNase-free DNase was used as a template to synthesize cDNA using the RevertAid First Strand cDNA Synthesis kit (Thermo Fisher Scientific, Inc.) according to the manufacturer's protocol. RT-qPCR analysis was performed using SYBR Premix Ex Taq (Takara Bio, Inc.) and CFX096 (Bio-Rad Laboratories, Inc.) using the following thermocycling conditions: Initial denaturation at $95^{\circ} \mathrm{C}$ for $30 \mathrm{sec}$, followed by 50 cycles at $95^{\circ} \mathrm{C}$ for $5 \mathrm{sec}$ and $60^{\circ} \mathrm{C}$ for $30 \mathrm{sec}$ for annealing and elongation. Target gene expression levels were normalized to $18 \mathrm{~S}$ ribosomal (r)RNA and quantified using the $2^{-\Delta \Delta \mathrm{Cq}}$ method (24). The sequences of the primers used were as follows: DRP1 forward, 5'-CTACCACGCCCG ACTACTTG-3' and reverse 5'-TCCTCTATCCCGTTGACA CC-3'; DRP2 forward, 5'-CACAGCGAGGGAGACTTAGG-3' and reverse 5'-CAGGGACCTCTTCGTCCTCT-3'; DRP3 forward, 5'-CAAGACGCTGGATTTCGATGC-3' and reverse 5'-ACGGTCACTCTTGTCCTTGGG-3'; DRP4 forward, 5'-ATCAGTCGGGGTTCAGCCTAT-3' and reverse 5'-GGA GAGAGAGGTGATGTTGGA-3'; DRP5 forward, 5'-TAA GGAGGCACTGGATTTGG-3' and reverse 5'-GCCGAGATA CTGGACACGTT-3'; 18S rRNA forward, 5'-CAGCCACCC GAGATTGAGCA-3 and reverse 5'-TAGTAGCGACGGGCG GTGTG-3'; Nestin forward, 5'- AACAGCGACGGAGGTCTC TA-3' and reverse 5'- TTCTCTTGTCCCGCAGACTT-3'; and SOX2 forward, 5'-CAAGATGCACAACTCGGAGA-3' and reverse, 5'-CGGGGCCCGTATTTATAATC-3'.

Patient dataset analysis and gene set enrichment analysis (GSEA). Microarray datasets from The Cancer Genome Atlas-GBM/low grade glioma (TCGA-GBM/LGG) database of the National Cancer Institute (https://cancergenome. nih.gov) and Gene Expression Omnibus (https://www.ncbi. nlm.nih.gov/geo/) were collected and classified into the 'DRP5-high' or 'DRP5-low' groups according to the higher or lower than average expression values. GSE4536 is a gene expression dataset comprising serum-free and serum-cultured GBM cells, whereas GSE67089 is a PN- and MES-subtype GSC gene expression dataset $(25,26)$. A total of five stemness-associated gene sets for GSEA were downloaded from MSigDB (https://www.gsea-msigdb.org/gsea/msigdb/index. jsp): 'Kyoto Encyclopedia of Genes and Genomes_Notch signaling pathway', 'Hallmark_Hedgehog signaling pathway', 'Hallmark_Wnt/ $\beta$-catenin signaling', 'Hallmark_IL6_JAK_ STAT3 signaling' and 'Hallmark_TNFA_VIA_NFKB signaling'. GSEA was performed using the GenePattern public 
server (https://cloud.genepattern.org) and the aforementioned datasets. GSEA was run using default options except for the collapse dataset set as 'False'.

Statistical analysis. Single sample GSEA (ssGSEA; ssGSEAProjection v10.0.3; https://www.genepattern.org/) was performed to obtain enrichment scores for PN, MES and GSC-high gene sets in each patient from the TCGA-GBM database. Kaplan-Meier survival analyses were performed using GraphPad Prism 6 (GraphPad Software, Inc.). Subsequently, Pearson's correlation coefficient (r value) was determined by comparing each enrichment score with the level of DRP5 expression. For statistical significance, all analyses were performed using a two-tailed unpaired Student's t-test, except for comparison of DRP5 expression with tumor grade and histology, for which a one-way ANOVA followed by the Tukey-Kramer test was used for multiple comparisons. Student's t-test was performed using Microsoft Office Excel (Microsoft Corporation) and ANOVA was performed using GraphPad Prism 6. Data were presented as the mean \pm standard error of the mean. $\mathrm{P}<0.05$ was considered to indicate a statistically significant difference.

\section{Results}

DRP5 identification and specific expression in GSCs. The GSE4536 and GSE67089 datasets (GBM patient-derived cancer stem cell microarray dataset) were analyzed to identify a gene list unique to GSCs. The data were compared with gene expression levels of non-GSCs and NHAs, and the gene list was classified into 'GSC-high' and 'GSC-low' groups ( $\mathrm{P}<0.05$; Fig. 1A). Genes in the GSC-high group were analyzed to identify GSC-specific genes based on the prognosis of patients and mRNA expression levels from TCGA-GBM/LGG microarray database. DRP5 was chosen as a predicted GSC-specific gene due to its high mRNA expression in GSCs and in high grade and aggressive brain tumors (Fig. 1B). Furthermore, the prognosis of patients with GBM with high DRP5 expression was lower than that in patients with low DRP5 expression (Fig. 1C).

To further analyze DRP5 expression in GSCs, RT-qPCR and western blotting were performed in GSCs and non-GSCs compared with NHAs (Fig. 1D and E, respectively). The results demonstrated that mRNA and protein expression of DRP5 in GSCs was elevated compared with non-GSCs and NHAs. Among GSCs, the PN-subtype GSCs (528NS, GSC11 and GSC23) exhibited higher DRP5 expression compared with the MES-subtype GSCs (GSC20) and the non-defined GSCs (GSC28). In addition, A172 non-GSC GBM cells exhibited similar expression levels of DRP5 compared with GSC11, but protein expression of DRP5 in A172 cells was markedly lower than those in the PN-subtype GSCs (Fig. 1D and E).

DRP5 was mainly localized in the nucleus of GSCs (Fig. 1F). A previous study reported that a carboxyl-terminal truncated isoform of DRP5 is localized to the nucleus compared with a full-length DRP5 that is localized in the cytoplasm and enhances cancer cell proliferation (27). It was therefore hypothesized that DRP5 in GSCs may be a carboxyl-terminal truncated isoform with biological functions that differ from the ones of full-length DRP5.
DRP5 is structurally and functionally distinct from other $D R P s$. It was hypothesized that DRP5 functions by interacting with other DRPs in GSCs, suggesting that DRPs might function as a complex (28). DRPs have a conserved D-hydantionase dihydropyrimidinase (D-HYD) domain; however, the amino acid sequence of DRP5 is different to that of the other DRPs (Fig. 2A). Although expression levels of DRP1-4 were higher in some GSCs and in A172 cells than those in NHA cells, high expression levels of DRP1-4 in patients with GBM were not associated with poor prognosis compared with low expression levels (Fig. 2B and C). A previous study reported that DRP5 has several amino acid substitutions at the core catalytic D-HYD domain and does not possess dihydropyrimidinase enzymatic activity (29). It was therefore hypothesized that DRP5 may serve a specific role in GSCs.

DRP5 maintains GSC properties. To investigate the role of DRP5 in GSCs, shRNA-mediated KD of DRP5 was performed in 528NS GSCs, since DRP5 expression was higher in PN-GSCs than in other GSCs. Following knockdown, the effect of DRP5 loss was evaluated on cancer stemness and tumorigenesis. DRP5 KD was confirmed in 528NS GSCs by western blotting (Fig. 3A). Subsequently, an LDA was performed to compare the tumor sphere-forming ability between GSCs lacking DRP5 and control cells. The results demonstrated that DRP5 KD significantly decreased the sphere-forming ability (Fig. 3B). Additionally, RT-qPCR was performed to determine whether DRP5 KD decreased the expression levels of GSC markers, such as Nestin and SRY-box transcription factor 2 (SOX2). The results revealed that the expression levels of Nestin and SOX2 were decreased in 528NS-DRP5 KD cells compared with control cells (Fig. 3C). Subsequently, in vivo experiments using a xenograft mouse model were performed, and 528NS-puro and 528NS-DRP5 KD cells were orthotopically injected into the brains of immunodeficient mice to investigate tumorigenicity. The results demonstrated that 528NS-DRP5 KD cells displayed decreased tumorigenicity compared with 528NS-puro cells, according to the tumor size evaluated following H\&E staining (Fig. 3D). Taken together, these findings suggested the importance of DRP5 in maintaining GSC stemness and tumorigenicity.

DRP5 expression is positively correlated with stemnessassociated gene signatures. GSEA was performed to confirm the associations between DRP5 expression patterns in the patient dataset of TCGA-GBM/LGG and cancer stemness-associated gene signatures. There are five known stemness-associated gene signatures: Notch, Hedgehog, Wnt/ $\beta$-catenin, Janus kinase-signal transducer and activator of transcription 3 (JAK-STAT3) and NF-кB. An enrichment plot for each signature was performed and the enriched signature in the DRP5-high patient group was analyzed. The Notch, Hedgehog and Wnt/ $\beta$-catenin signatures were enriched in the DRP5-high group, whereas the JAK-STAT3 and NF- $\kappa \mathrm{B}$ signatures were enriched in the DRP5-low group (Fig. 4A). Tumor necrosis factor- $\alpha$ (TNF- $\alpha$ )/NF- $\kappa B$ signaling lead to a PN-MES transition, resulting in the enrichment of CD44 subpopulations and radio-resistant phenotypes (30). Additionally, JAK-STAT3 signaling has previously been 

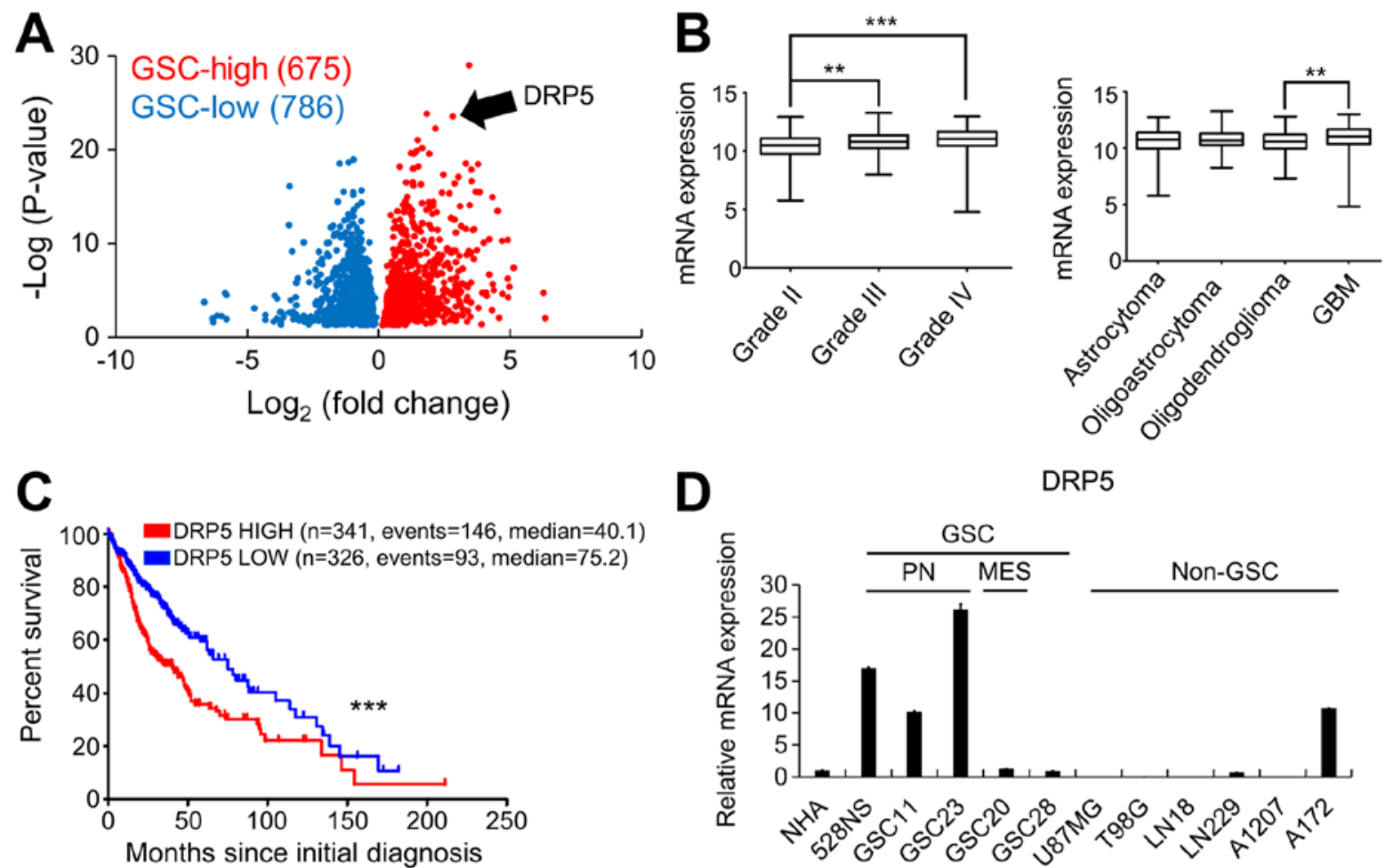

DRP5
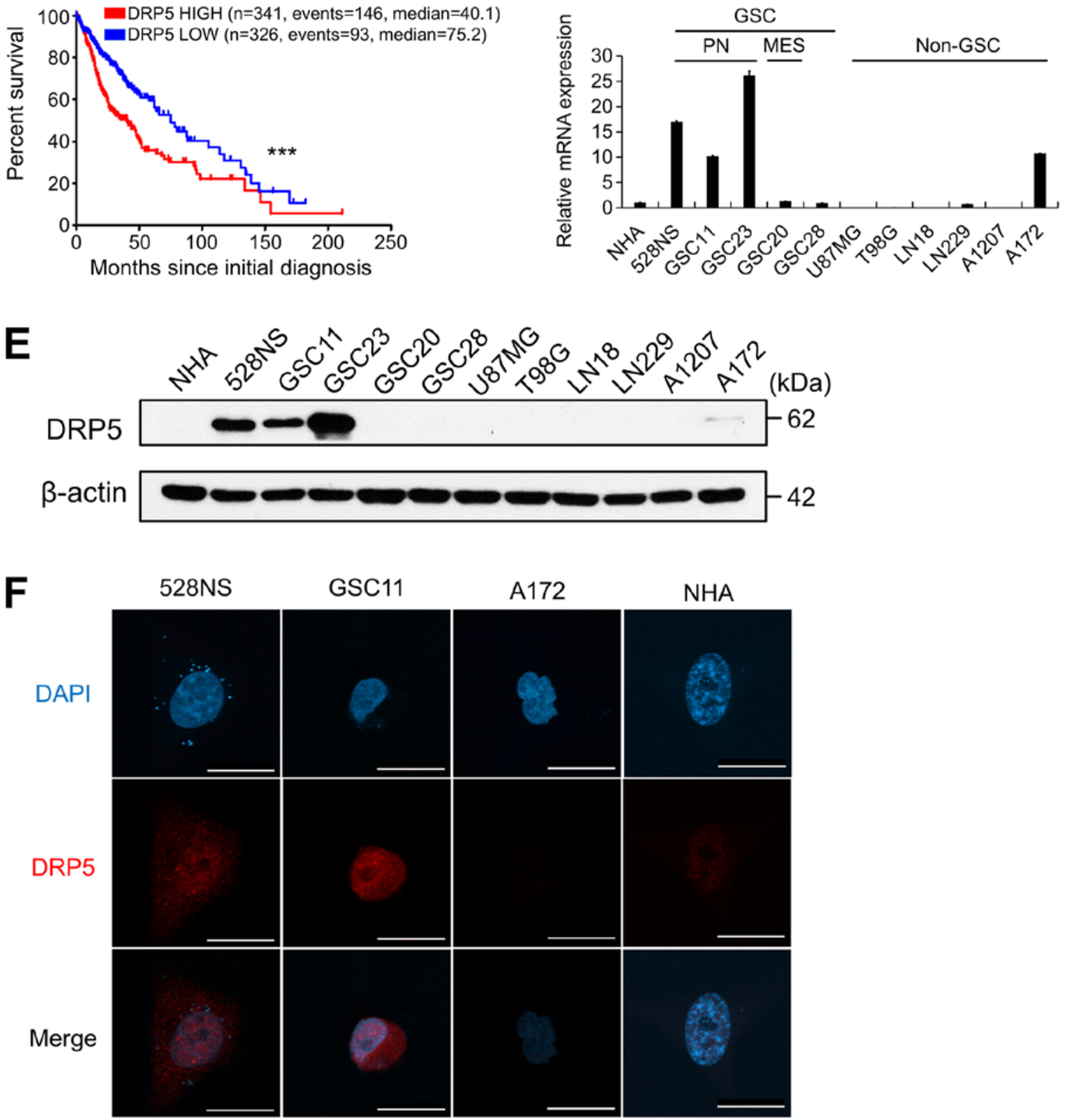

Figure 1. DRP5 expression in GSCs and its clinical significance. (A) Identification of DRP5 as a GSC-specific upregulated gene using GSE4536 and GSE67089 datasets. GSC-high and GSC-low cut-off values are \pm 2 -fold change. (B) DRP5 expression in patients in TCGA-GBM/LGG according to tumor grade and histology. (C) Kaplan-Meier survival analysis of patients in TCGA-GBM/LGG according to the expression levels of DRP5. (D) Reverse transcription-quantitative PCR analysis analyzing relative DRP5 mRNA expression in PN- and MES-subtype GSCs and non-GSC glioma cells compared with NHAs. Data were analyzed using the $2^{-\triangle \Delta C q}$ method, and human $18 \mathrm{~S}$ ribosomal RNA was used as the internal control. (E) Western blot analysis of DRP5 protein in GSCs and non-GSC glioma cells compared with NHAs. Human $\beta$-actin was used as the loading control. (F) Immunofluorescence images displaying DRP5 (red) in NHAs, two GSCs (528N and GSC11), and the non-GSC A172 glioma cells. Scale bar, $20 \mu \mathrm{m} .{ }^{* *} \mathrm{P}<0.01 ;{ }^{* * *} \mathrm{P}<0.001$. DRP5, dihydropyrimidinase-related protein 5; GBM, glioblastoma; GSC, GBM stem cell; TCGA, The Cancer Genome Atlas; PN, proneural; MES, mesenchymal; NHA, normal human astrocytes; DAPI, 4',6-diamidino-2-phenylindole. 
A

DRP5 (564 aa)

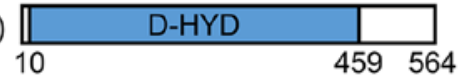

DRP1 (686aa)

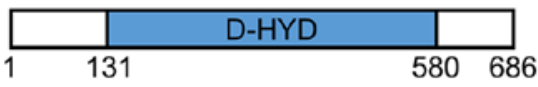

DRP2 (678 aa)

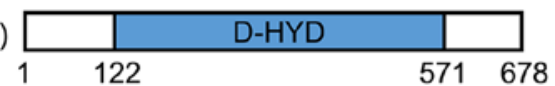

DRP3 (684 aa)

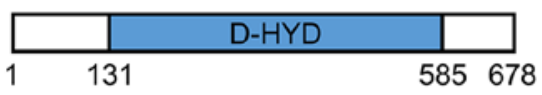

DRP4 (572 aa)

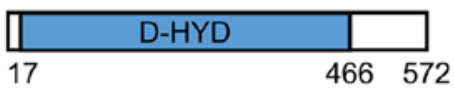

B

응
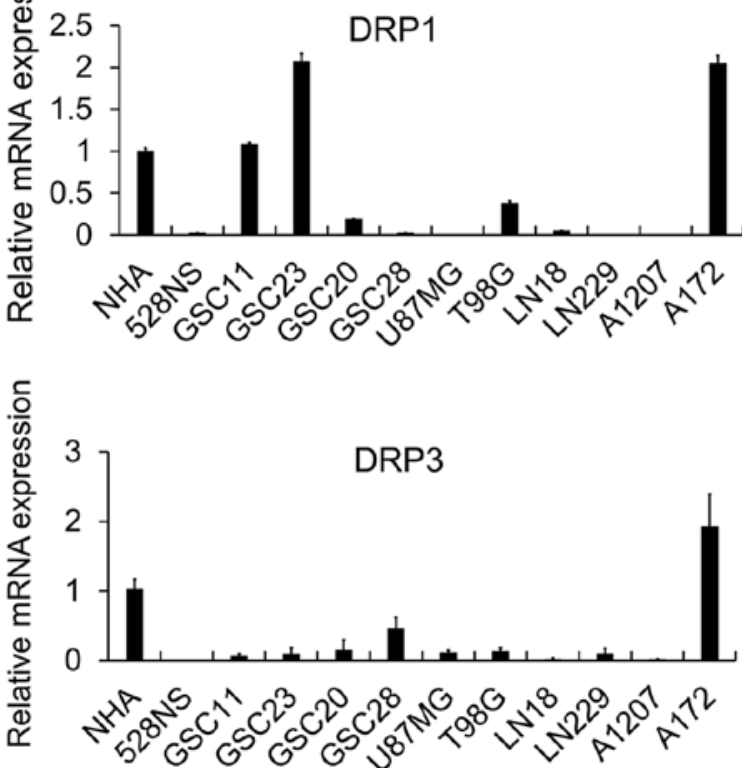

C
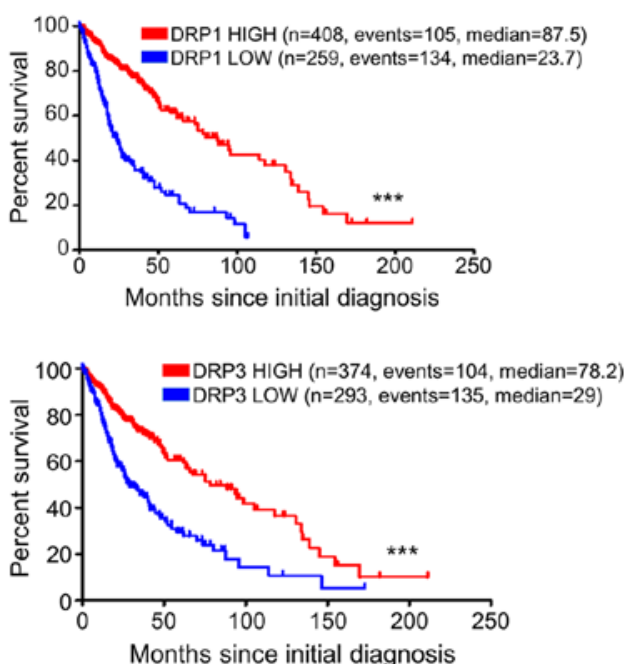

\begin{tabular}{|l|c|c|c|c|c|}
\hline & DRP5 & DRP4 & DRP3 & DRP2 & DRP1 \\
\hline DRP5 & - & $50 \%$ & $52 \%$ & $52 \%$ & $51 \%$ \\
\hline DRP4 & $51 \%$ & - & $69 \%$ & $75 \%$ & $69 \%$ \\
\hline DRP3 & $54 \%$ & $73 \%$ & - & $69 \%$ & $69 \%$ \\
\hline DRP2 & $54 \%$ & $77 \%$ & $78 \%$ & - & $68 \%$ \\
\hline DRP1 & $53 \%$ & $71 \%$ & $78 \%$ & $77 \%$ & - \\
\hline
\end{tabular}
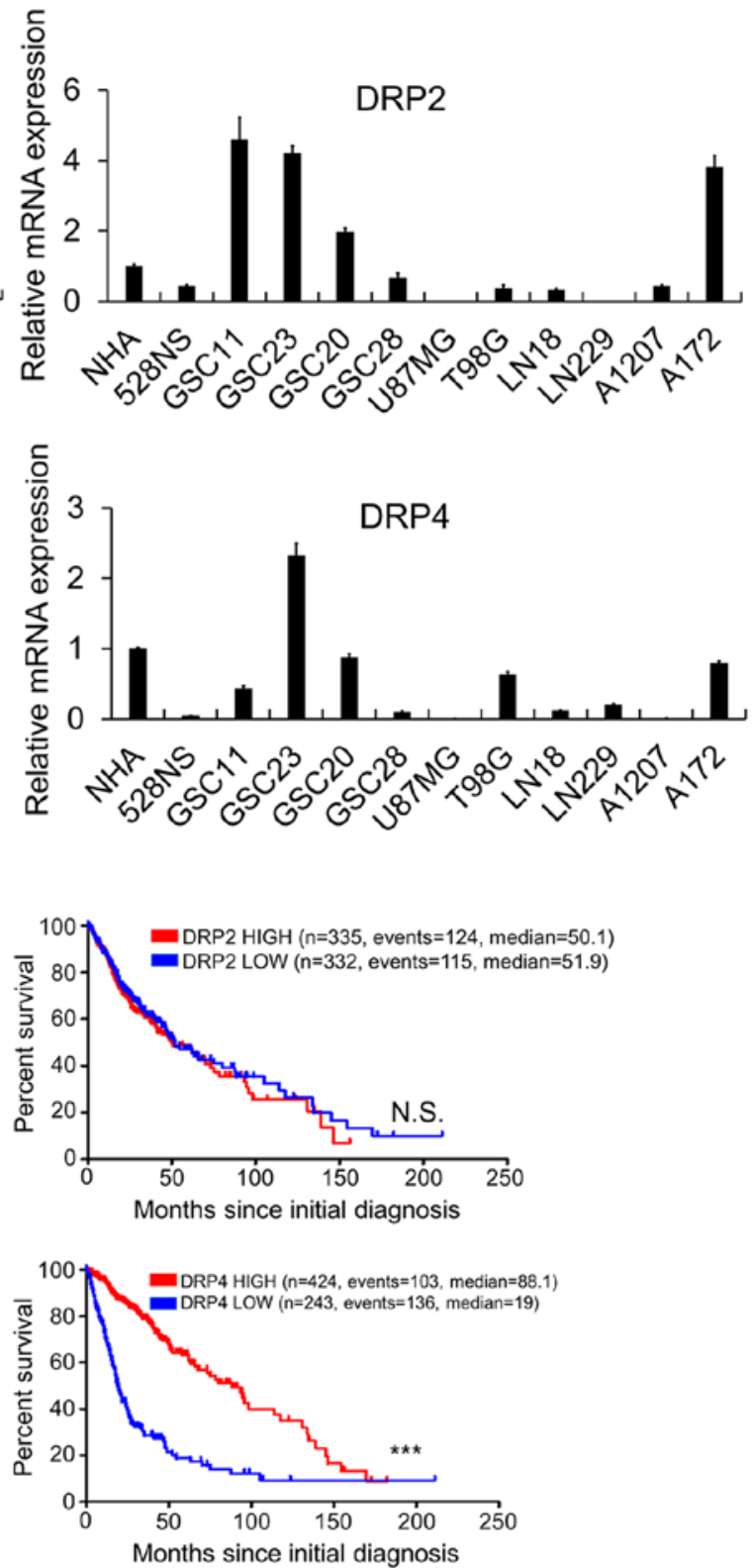

Figure 2. DRP5 displayed different gene characteristics compared with DRP1-4 genes. (A) Amino acid sequences of DRP1-5 and their conserved D-HYD domains. Right panel presents the DRP family amino acid sequence similarities. Black-labeled percentages are whole amino sequence similarities; blue-labeled percentages are D-HYD domain sequence similarities. (B) Reverse transcription-quantitative PCR analysis comparing the relative DRP1-4 mRNA expression in GSCs and non-GSC glioma cells with those in normal human astrocytes. Data were analyzed using the $2^{-\Delta \Delta \mathrm{Cq}}$ method, and human 18S ribosomal RNA was used as the internal control. (C) Kaplan-Meier survival analysis of patients in The Cancer Genome Atlas-GBM/LGG according to the expression levels of DRP1-4. ${ }^{* * *} \mathrm{P}<0.001$. NS, not significant; D-HYD, D-hydantionase dihydropyrimidinase; DRP, dihydropyrimidinase-related protein; GBM, glioblastoma; GSC, GBM stem cell. 
A
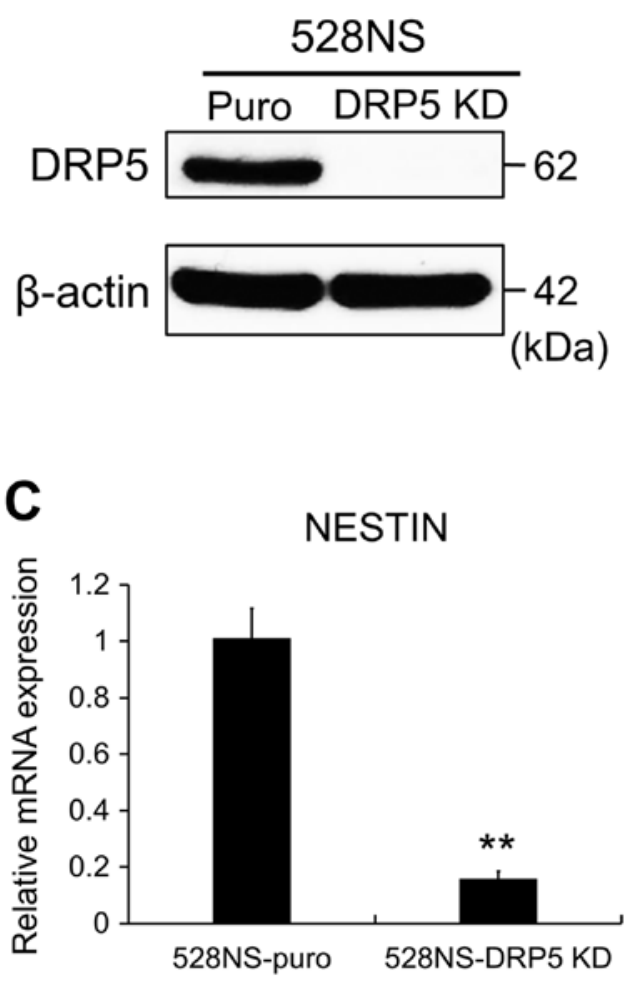

B
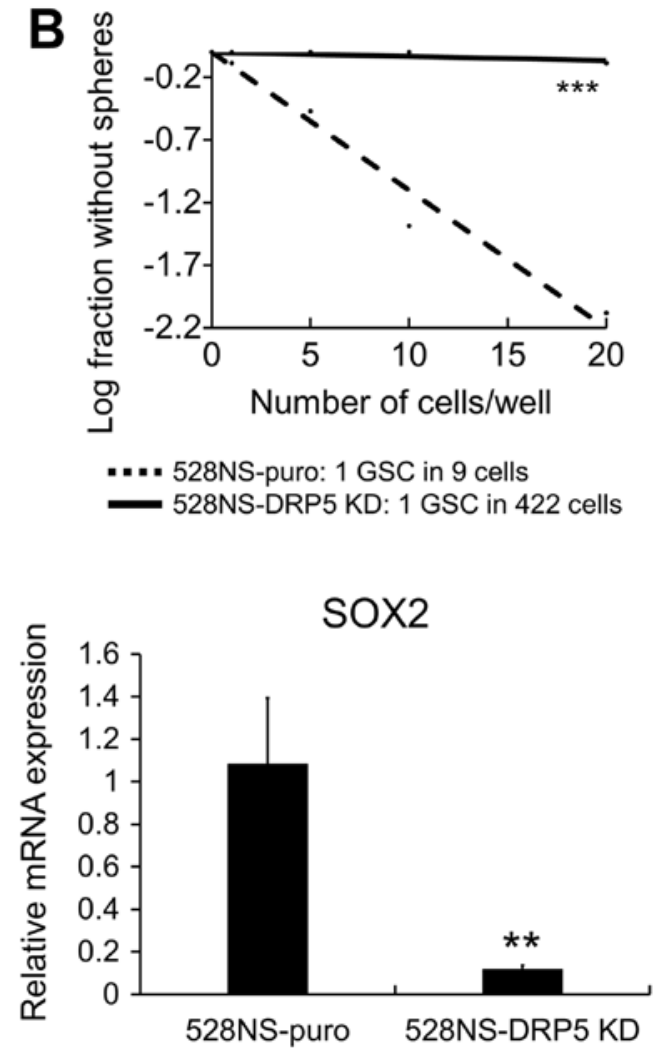

\section{528NS-Puro}

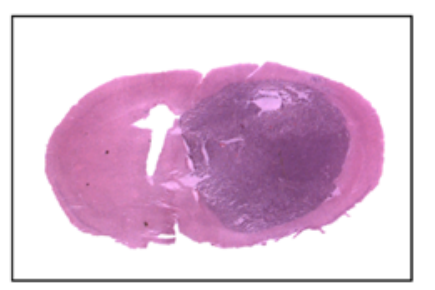

528NS-DRP5 KD

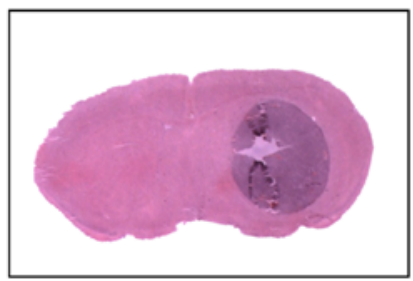

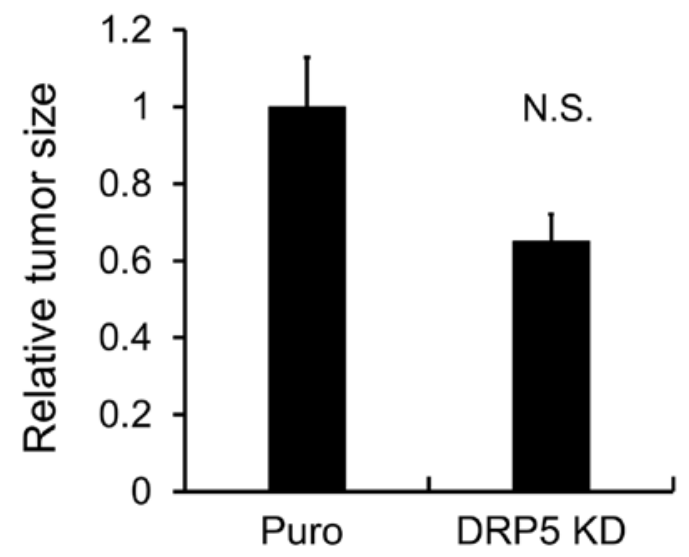

Figure 3. DRP5 retains GSC properties and tumorigenicity. (A) Western blot analysis of DRP5 protein in 528NS-DRP5 KD and control (Puro) cells. Human $\beta$-actin was used as the loading control. (B) Limiting dilution assay analyzing 528NS neurosphere formation following DRP5 KD. Experiment was performed in duplicate. (C) Reverse transcription-quantitative PCR analysis of the GSC markers Nestin and SOX2 in 528NS-DRP5 KD and control (Puro) cells. Experiment was performed in triplicate. (D) Hematoxylin and eosin staining of representative xenograft brain tumors by orthotopic injection of 528NS-DRP5 KD ( $n=5)$ and control (Puro) cells $(n=5)$. The right panel indicates the average tumor size. ${ }^{* *} \mathrm{P}<0.01 ;{ }^{* * *} \mathrm{P}<0.001$. NS, not significant; KD, knockdown; SOX2, SRY-box transcription factor 2; DRP5, dihydropyrimidinase-related protein 5; GSC, glioblastoma stem cell.

associated with MES differentiation and poor clinical outcomes (31). Therefore, NF- $\kappa \mathrm{B}$ and JAK-STAT3 signatures are considered MES-subtype stemness signatures. The present results revealed that DRP5 expression was positively correlated with PN-subtype stemness signatures and negatively correlated with MES-subtype signatures (Fig. 4B). PN-gene signatures were positively correlated with Notch, Hedgehog and $\mathrm{Wnt} / \beta$-catenin signatures, whereas MES-gene signatures were positively correlated with $\mathrm{NF}-\kappa \mathrm{B}$ and JAK-STAT3 signatures (Fig. 4C). Furthermore, DRP5 expression was positively correlated with the GSC-high gene list in patients with GBM (Fig. 4D). Overall, these results suggested that DRP5 may be considered as a novel biomarker of PN-subtype GSCs.

\section{Discussion}

GSCs are resistant to chemotherapy and radiotherapy and promote tumor growth following surgical resection of the 

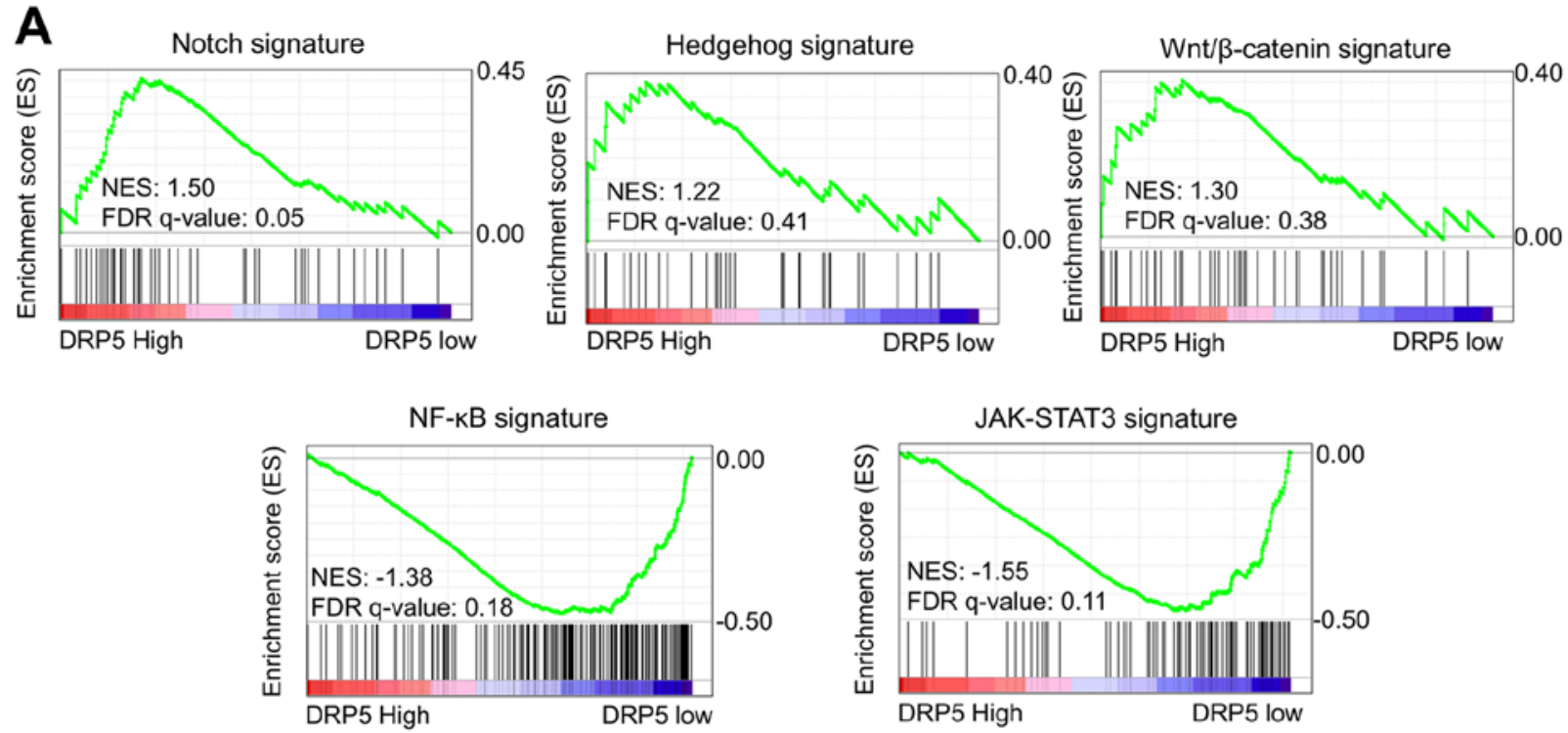

\section{B DRP5 \& proneural correlation}
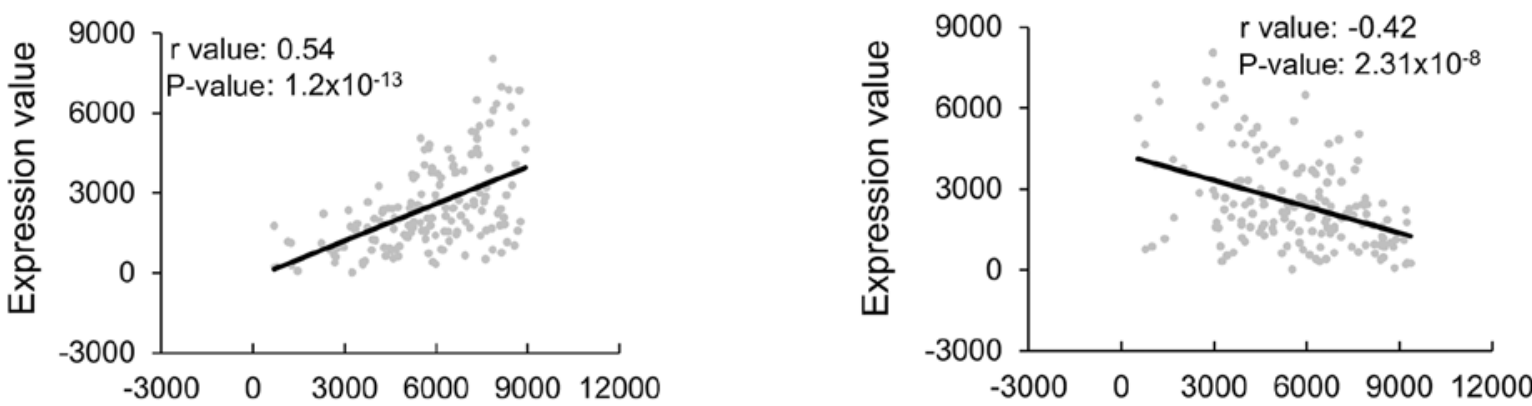

Enrichment score (ES)

Enrichment score (ES)

C

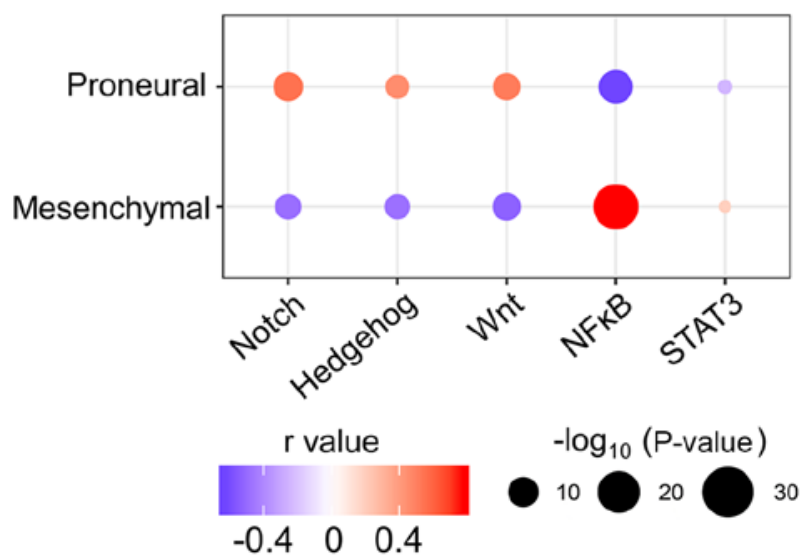

D

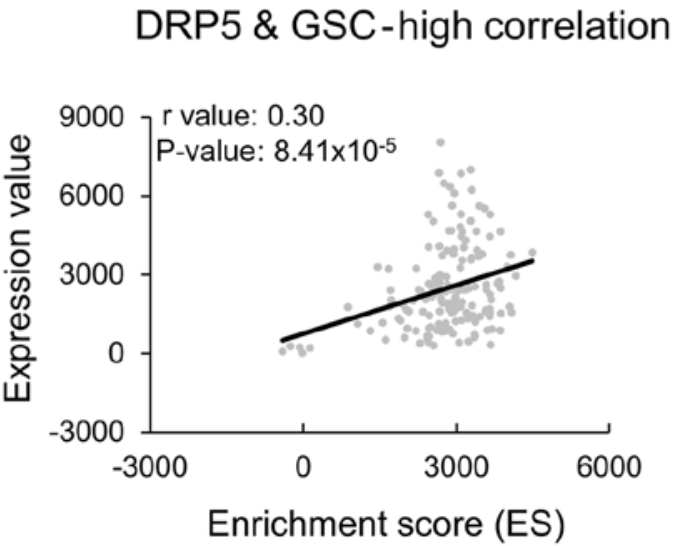

Figure 4. DRP5 expression correlates with stemness-associated gene signatures. (A) Gene set enrichment analysis enrichment plots presenting Notch, Hedgehog and Wnt/ $\beta$-catenin enrichment in patients with DRP5-high GBM ( $\mathrm{n}=57$ ). JAK-STAT3 and NF- $\kappa$ B enrichment in patients with DRP5-low GBM ( $=89$ ). (B) Correlation plots between DRP5 expression and proneural (left) or mesenchymal (right) gene signature in patients with GBM ( $\mathrm{n}=146$ ). (C) Correlation plots between stemness gene signatures and proneural or mesenchymal gene signatures. (D) Correlation plot between GBM stem cell-high gene signature and DRP5 expression in patients with GBM (n=146). JAK-STAT3, Janus kinase- signal transducer and activator of transcription 3; DRP5, dihydropyrimidinase-related protein 5; GBM, glioblastoma; NES, normalized enrichment score; FDR, false discovery rate.

tumor (6-9). GSC subtype has been associated with treatment efficacy and prognosis of patients (13). Numerous strategies have been developed to eradicate GSCs; however, they have proven ineffective (32). The failure of these therapies can partly be explained by the different drug responses depending on the GSC subtype (33). In order to development successful 
treatment options, it is therefore essential to properly define the GSC subtype of the cancer.

The present study identified DRP5 using gene expression database analysis from GBM patient-derived differentiated and stem-like cancer cells. Notably, DRP5 displayed PN-GSC-specific expression, and the prognosis of patients from TCGA-GBM/LGG dataset with high DRP5 expression was lower compared with patients with low DRP5 expression. However, in TCGA-GBM dataset, DRP5 expression was not significant in determining patient prognosis (data not shown). DRP5 may therefore be only one of the factors that maintain stemness signature, making it hard to explain how DRP5 expression could determine the aggressiveness of GBM $(34,35)$. However, several studies reported that a single factor can affect the progression of low-grade glioma (36-38). Therefore, high DPR5 expression in PN-GSCs may promote the malignancy of brain tumors. Furthermore, DRP5 KD decreased tumorigenicity and numerous stemness-associated molecular signaling pathways of GSCs, although the tumor size in mice with DRP5 KD was not statistically different from that in control mice. In a subcutaneous mouse cancer model, tumor size is crucial for determining tumorigenicity; however, in an orthotopic brain cancer model, tumor size is not the only determinant of tumorigenicity. The major cause of death in brain tumor mouse models is the development of neurodegenerative disorders due to increased brain pressure and tumor infiltration in neighboring nervous tissues (39). Overall, DRP5 seems to maintain GSC characteristics and may therefore be considered as a functional biomarker of PN-GSCs.

DRP5 is a cytosolic protein involved in normal neuronal development, including neurite outgrowth and axon guidance. Dihydropyrimidinase (DHPase) is an enzyme that degrades pyrimidines, such as uracil and thymine (40). The D-HYD domain is therefore required for successful enzymatic activity. In the DRP family, except DHPase, the enzymatic catalytic core amino acids of the D-HYD domain are substituted, abrogating its enzymatic activity (29). The DRP family is therefore functionally different from DHPase. DRP5 interacts with other DRP family genes, including DRP2, DRP3 and DRP4 (41). However, in the GSC lines used in the present study, especially 528NS, the expression levels of other DRP family genes were lower than that of DRP5. Therefore, DRP5 may function alone to regulate the characteristics of GSCs. Although the truncated form of DRP5 is reported to be translocated to the nucleus in GBM cells and to promote cell proliferation, the underlying mechanism of this phenomenon remains unclear (26). The present results demonstrated that DRP5 was specifically expressed in PN-GSCs and was localized to the nucleus. Therefore, nuclear DRP5 may promote cell proliferation and maintain PN-GSC characteristics. The mechanism of DRP5 translocation to the nucleus and the role of nuclear DRP5 require further investigation. A previous study has demonstrated that DRP5 regulates stemness-associated molecular signaling pathways, such as the Notch signaling pathway (20). In addition, the current study revealed that DRP5 expression was positively associated with Notch, Hedgehog and Wnt/ $\beta$-catenin signaling pathways. DRP5 in the nucleus may therefore influence gene expression by acting as a transcriptional regulator, thereby regulating GSC stemness and tumorigenicity.
In conclusion, the results from the present study demonstrated that DRP5 was specifically expressed in the PN-subtype of GSCs, suggesting that it may be used as a functional biomarker of GBM derived from PN-GSCs.

\section{Acknowledgements}

The authors would like to thank Dr. Man Bock Gu, the director of BK21 PLUS for Biotechnology in the Department of Biotechnology at Korea University, for the support of publication cost.

\section{Funding}

The present study was supported by the National Research Foundation (grant no. 2017R1E1A1A01074205).

\section{Availability of data and materials}

The datasets used and/or analyzed during the current study are available from the corresponding author on request. GSE4536 (https://www.ncbi.nlm.nih.gov/geo/query/acc. cgi?acc=GSE4536) and GSE67089 (https://www.ncbi.nlm. nih.gov/geo/query/acc.cgi?acc=GSE67089) are available in the GEO database.

\section{Authors' contributions}

MGP, SS and HK conceived the study and the experiments. MGP and SWH performed the experiments. MGP and SHC captured and analyzed the staining images. MGP performed the statistical analysis. MGP and HK wrote and corrected the manuscript. All authors read and approved the final manuscript.

\section{Ethics approval and consent to participate}

Animal experiments were performed in the specificpathogen-free facility with the approval of the Korea University Institutional Animal Care \& Use Committee (approval no. KUIACUC-2018-0017) and according to the governmental and institutional guidelines and Korean regulations.

\section{Patient consent for publication}

Not applicable.

\section{Competing interests}

The authors declare that they have no competing interests.

\section{References}

1. Mazaris P, Hong X, Altshuler D, Schultz L, Poisson LM, Jain R, Mikkelsen T, Rosenblum M and Kalkanis S: Key determinants of short-term and long-term glioblastoma survival: A 14-year retrospective study of patients from the Hermelin Brain Tumor Center at Henry Ford Hospital. Clin Neurol Neurosurg 120: 103-112, 2014.

2. Porter KR, McCarthy BJ, Freels S, Kim Y and Davis FG: Prevalence estimates for primary brain tumors in the United States by age, gender, behavior and histology. Neuro Oncol 12: 520-527, 2010. 
3. Omuro A and DeAngelis LM: Glioblastoma and other malignant gliomas: A clinical review. JAMA 310: 1842-1850, 2013.

4. Dobelbower MC, Burnett Iii OL, Nordal RA, Nabors LB Hyatt MD and Fiveash JB: Patterns of failure for glioblastoma multiforme following concurrent radiation and temozolomide. J Med Imaging Radiat Oncol 55: 77-81, 2011.

5. Neman J and Jandial R: Decreasing glioma recurrence through adjuvant cancer stem cell inhibition. Biologics 4: 157-162, 2010.

6. Reya T, Morrison SJ, Clarke MF and Weissman IL: Stem cells, cancer, and cancer stem cells. Nature 414: 105-111, 2001.

7. Lathia JD, Mack SC, Mulkearns-Hubert EE, Valentim CL and Rich JN: Cancer stem cells in glioblastoma. Genes Dev 29: 1203-1217, 2015

8. Chen J, Li Y, Yu TS, McKay RM, Burns DK, Kernie SG and Parada LF: A restricted cell population propagates glioblastoma growth after chemotherapy. Nature 488: 522-526, 2012.

9. Xie Q, Mittal S and Berens ME: Targeting adaptive glioblastoma: An overview of proliferation an invasion. Neuro Oncol 16 : $1575-1584,2014$

10. Kanabur P, Guo S, Simonds GR, Kelly DF, Gourdie RG, Verbridge SS and Sheng Z: Patient-derived glioblastoma stem cells respond differentially to targeted therapies. Oncotarget 7 : 86406-86419, 2016.

11. Jackson M, Hassiotou F and Nowak A: Glioblastoma stem-like cells: At the root of tumor recurrence and a therapeutic target. Carcinogenesis 36: 177-185, 2015.

12. Wang Q, Hu B, Hu X, Kim H, Squatrito M, Scarpace L, deCarvalho AC, Lyu S, Li P, Li Y, et al: Tumor evolution of glioma-intrinsic gene expression subtypes associates with immunological changes in the microenvironment. Cancer Cell 32 42-56.e6, 2017.

13. Schmidt EF and Strittmatter SM: The CRMP family of proteins and their role in Sema3A signaling. Adv Exp Med Biol 600: 1-11, 2007.

14. Quach TT, Honnorat J, Kolattukudy PE, Khanna R and Duchemin AM: CRMPs: Critical molecules for neurite morphogenesis and neuropsychiatric diseases. Mol Psychiatry 20: $1037-1045,2015$

15. Charrier E, Reibel S, Rogemond V, Aguera M, Thomasset N and Honnorat J: Collapsin response mediator proteins (CRMPs) Involvement in nervous system development and adult neurodegenerative disorders. Mol Neurobiol 28: 51-64, 2003.

16. Tan F, Thiele CJ and Li Z: Collapsin response mediator proteins: Potential diagnostic and prognostic biomarkers in cancers (Review). Oncol Lett 7: 1333-1340, 2014.

17. Zheng Y, Sethi R, Mangala LS, Taylor C, Goldsmith J, Wang M, Masuda K, Karaminejadranjbar M, Mannion D, Miranda F, et al: Tuning microtubule dynamics to enhance cancer therapy by modulating FER-mediated CRMP2 phosphorylation. Nat Commun 9: 476, 2018.

18. Yang Y, Jiang Y, Xie D, Liu M, Song N, Zhu J, Fan J and Zhu C: Inhibition of cell-adhesion protein DPYSL3 promotes metastasis of lung cancer. Respir Res 19: 41, 2018.

19. Nagano H, Hashimoto N, Nakayama A, Suzuki S, Miyabayashi Y, Yamato A, Higuchi S, Fujimoto M, Sakuma I, Beppu M, et al: p53-inducible DPYSL4 associates with mitochondrial supercomplexes and regulates energy metabolism in adipocytes and cancer cells. Proc Natl Acad Sci USA 115: 8370-8375, 2018.

20. Moutal A, Honnorat J, Massoma P, Desormeaux P, Bertrand C, Malleval C, Watrin C, Chounlamountri N, Mayeur ME, Besancon R, et al: CRMP5 controls glioblastoma cell proliferation and survival through Notch-dependent signaling. Cancer Res 75: 3519-3528, 2015.

21. Wang L, Liu W, Tang H, Xie X, Zou C, Wang Y, Gao Z and Yin J: DRP5 is involved in cancer cell growth and predicts poor prognosis in human osteosarcoma. Cancer Med 6: 982-993, 2017.

22. Dubey D, Lennon VA, Gadoth A, Pittock SJ, Flanagan EP Schmeling JE, McKeon A and Klein CJ: Autoimmune CRMP5 neuropathy phenotype and outcome defined from 105 cases. Neurology 90: e103-e110, 2018.

23. Petit V, Massonnet G, Maciorowski Z, Touhami J, Thuleau A, Nemati F, Laval J, Cahteau-Joubert S, Servely JL, Vallerand D, et al: Optimization of tumor xenograft dissociation for the profiling of cell surface markers and nutrient transporters. Lab Invest 93: 611-621, 2013.
24. Livak KJ and Schmittgen TD: Analysis of relative gene expression data using real-time quantitative PCR and the 2(-Delta Delta C(T)) method. Methods 25: 402-408, 2001

25. Lee J, Kotliarova S, Kotliarov Y, Li A, Su Q, Donin NM, Pastorino S, Purow BW, Christopher N, Zhang W, et al: Tumor stem cells derived from glioblastomas cultured in bFGF and EGF more closely mirror the phenotype and genotype of primary tumors than do serum-cultured cell lines. Cancer Cell 9: 391-403, 2006.

26. Mao P, Joshi K, Li J, Kim SH, Li P, Santana-Santos L, Luthra S, Chandran UR, Benos PV, Smith L, et al: Mesenchymal glioma stem cells are maintained by activated glycolytic metabolism involving aldehyde dehydrogenase $1 \mathrm{~A} 3$. Proc Natl Acad Sci USA 110: 8644-8649, 2013

27. Brot S, Malleval C, Benetollo C, Auger C, Meyronet D, Rogemond $\mathrm{V}$, Honnorat $\mathrm{J}$ and Moradi-Ameli M: Identification of a new CRMP5 isoform present in the nucleus of cancer cells and enhancing their proliferation. Exp Cell Res 319: 588-599, 2013.

28. Tan M, Cha C, Ye Y, Zhang J, Li S, Wu F, Gong S and Guo G: CRMP4 and CRMP2 interact to coordinate cytoskeleton dynamics, regulating growth cone development and axon elongation. Neural Plast 2015: 947423, 2015.

29. Ponnusamy R and Lohkamp B: Insights into the oligomerization of CRMPs: Crystal structure of human collapsin response mediator protein 5. J Neurochem 125: 855-868, 2013.

30. Bhat KPL, Balasubramaniyan V, Vaillant B, Ezhilarasan R, Hummelink K, Hollingsworth F, Wani K, Heathcock L, James JD, Goodman LD, et al: Mesenchymal differentiation mediated by $\mathrm{NF}-\kappa \mathrm{B}$ promotes radiation resistance in glioblastoma. Cancer Cell 24: 331-346, 2013.

31. Carro MS, Lim WK, Alvarez MJ, Bollo RJ, Zhao X, Snyder EY, Sulman EP, Anne SL, Doetsch F, Colman H, et al: The transcriptional network for mesenchymal transformation of brain tumours. Nature 463: 318-325, 2010.

32. Barbato L, Bocchetti M, Di Biase A and Regad T: Cancer stem cells and targeting strategies. Cells 8: E926, 2019.

33. Kwon SM, Kang SH, Park CK, Jung S, Park ES, Lee JS, Kim SH and Woo HG: Recurrent glioblastomas reveal molecular subtypes associated with mechanistic implications of drug-resistance. PLoS One 10: e0140528, 2015

34. Dagogo-Jack I and Shaw AT: Tumor heterogeneity and resistance to cancer therapies. Nat Rev Clin Oncol 15: 81-94, 2018.

35. Patel AP, Tirosh I, Trombetta JJ, Shalek AK, Gillespie SM, Wakimoto H, Cahill DP, Nahed BV, Curry WT, Martuza RL, et al: Single-cell RNA-seq highlights intratumoral heterogeneity in primary glioblastoma. Science 344: 1396-1401, 2014.

36. Dai X, Liao K, Zhuang Z, Chen B, Zhou Z, Zhou S, Lin G, Zhang F, Lin Y, Miao Y, et al: AHIF promotes glioblastoma progression and radioresistance via exosomes. Int J Oncol 54: 261-270, 2019.

37. Qian Z, Ren L, Wu D, Yang X, Zhou Z, Nie Q, Jiang G, Xue S, Weng W, Qiu Y and Lin Y: Overexpression of FoxO3a is associated with glioblastoma progression and predicts poor patient prognosis. Int J Cancer 140: 2792-2804, 2017.

38. Luo $\mathrm{K}$ and Zhuang K: High expression of PCBP2 is associated with proression and poor prognosis in patients with glioblastoma. Biomed Pharmacother 94: 659-665, 2017.

39. Martínez-Murillo R and Martínez A: Standardization of an orthotopic mouse brain tumor model following transplantation of CT-2A astrocytoma cells. Histol Histopathol 22: 1309-1326, 2007.

40. Huang YH, Ning ZJ and Huang CY: Crystal structure of dihydropyrimidinase in complex with anticancer drug 5-fluorouracil. Biochem Biophys Res Commun 519: 160-165, 2019.

41. Fukada M, Watakabe I, Yuasa-Kawada J, Kawachi H, Kuroiwa A, Matsuda A and Noda M: Molecular characterization of CRMP5, a novel member of the collapsin response mediator protein family. J Biol Chem 275: 37957-37965, 2000.

This work is licensed under a Creative Commons Attribution-NonCommercial-NoDerivatives 4.0 International (CC BY-NC-ND 4.0) License. 